\title{
Féminité et sorcellerie chez les théoriciens de la démonologie à la fin du Moyen Age: Etude du Malleus Maleficarum*
}

\author{
ELIANE CAMERLYNCK
}

C'est au XVe siècle, dans le cadre d'une société aux prises avec le retour offensif de la Peste Noire, la guerre de Cent Ans, l'avance turque, le grand Schisme, les excommunications, les soulèvements et les guerres que fut publié en 1486 ou 1487 le Malleus Maleficarum de Jacques Sprenger et Henri Institoris.

Le Malleus Maleficarum, destiné aux inquisiteurs, aux juges civils et ecclésiastiques, ne représente qu'un exemple particulier dans l'ensemble de la littérature traitant des phénomènes de sorcellerie. En effet, on est frappé par la prolifération de ces publications du $\mathrm{XIV}^{\mathrm{e}}$ au XVII ${ }^{\mathrm{e}}$ siècle, qui reflète - en partie - l'ébranlement des esprits face à une "dangereuse conjonction de peurs" qui, selon Jean Delumeau, auraient assailli la civilisation européenne à partir du XIV siècle. $^{1}$

Ainsi que l'auteur le précise, il y a eu dynamisme mais aussi angoisse face à une situation extérieure périlleuse et à des transformations d'ordre intérieur au sein même des Etats: montée de l'absolutisme, dépression démographique, repli agricole dû à la détérioration des conditions climatiques depuis 1300 , maintenant les populations en état permanent "d'alerte biologique." Les malheurs collectifs et individuels eurent comme effet de provoquer des colères sans but, des gestes aberrants, une propension à la morbidité, un effondrement des structures familiales, une angoisse chronique - bref, un sentiment d'impuissance et d'incompréhension.

Les comportements collectifs en temps de peste ont déjà été analysés ainsi que les effets des violentes décharges d'agressivité qui accompagnent l'épidémie sans oublier la recherche des responsables du fléau. Tout individu mal intégré, mal assimilé, était un coupable potentiel qu'il fût étranger, voyageur, lépreu, hérétique ou sorcier. Ces "marginaux" ne pouvaient être que des complices de Satan. ${ }^{2}$

Cette peur du diable, de sa présence diffuse parmi les hommes, des pièges et perversions qu'il propageait, des fantasmes qu'il engendrait, mobilisa

* Communication présentée en mai 1981 au Congrès des Sociétés savantes (Société canadienne d'études de la Renaissance). 
l'élite politique, intellectuelle et religieuse pendant toute la période constituant la charnière entre la fin du Moyen Age et les Temps Modernes. Les théologiens, juristes, écrivains et démonologues les plus zélés allaient démasquer Satan dans leurs ouvrages et persuader les sceptiques de la dangereuse réalité des entreprises du Malin. ${ }^{3}$

Les inquisiteurs et les juges devaient être guidés dans leur mission d'épuration; en effet, il était indispensable non seulement d'être à l'affût des rumeurs et des dénonciations, d'épier les coupables et de les incarcérer, mais bien plus, de les isoler, de les questionner, de les torturer puis de les livrer au supplice du feu après avoir obtenu l'essentiel: l'aveu.

Dans le cadre du sujet qui nous intéresse, il est donc important d'insister sur cette poussée du satanisme qui se manifesta à la fin du Moyen Age et qui engendra une mentalité obsidionale, provoquant la mobilisation de toutes les énergies contre "l'offensive démoniaque." La contre-offensive théorique des milieux dirigeants et des prédicateurs se fit l'écho de l'offensive démoniaque. $\mathrm{Au} \mathrm{XV}$ 'iècle, elle s'acharna sur les hommes et surtout sur les femmes accusés de sorcellerie.

C'est dans ce contexte que la bulle d'Innocent VIII Summis desiderantes affectibus $^{4}$ promulguée en décembre 1484, autorisa Jacques Sprenger et Henri Institoris - dominicains inscrits à l'Ordre des Frères Prêcheurs - de poursuivre leur mission inquisitoriale dans la région du Haut Rhin, protégea leur cause, confirma leur compétence juridique, les assura de l'appui de l'évêque de Strasbourg et leur donna le plein pouvoir de s'attaquer aux sorcières en Allemagne. Cette bulle stimula le zèle des auteurs qui publièrent deux ans plus tard (vers 1486) leur "guide particulier spécialisé dans la poursuite de l'hérésie de sorcellerie et de magie diabolique," le Malleus Maleficarum. ${ }^{5}$

Fondateur d'une confrérie du Rosaire, théologien connu et considéré, professeur réputé de l'Université de Cologne, Jacques Sprenger (1436-1495) n'aurait pas participé très activement à la rédaction du Malleus. ${ }^{6}$

Par contre, Henri Institoris (1430-1505) fut non seulement un inquisiteur zélé dans les diocèses de Constance et Brixen, dans la province de Teutonie, en Moravie et en Bohême, mais aussi le principal auteur du Malleus Maleficarum, guide pour Inquisiteurs, prédicateurs, juges civils et ecclésiastiques, destiné à définir, surveiller, convaincre, guérir et punir. ${ }^{7}$

Outil de combat, il avait comme mission d'écraser les "malfaisantes." L'utilisation du terme maleficarum constituait une innovation désignant plus spécialement cette hérésie des sorcières du $\mathrm{XV}^{\mathrm{e}}$ siècle. En employant cette désignation, les auteurs ont voulu insister sur les délits d'ordre matériel occasionnés par les sorcières plutôt que sur l'injustice des actes hérétiques d'ordre spirituel afin d'éveiller l'attention des juges séculiers. ${ }^{8}$ De plus, l'utilisation du féminin (maleficarum) indique que dans la pensée de l'auteur, cette hérésie frappait plus souvent les femmes que les hommes: "pourquoi ce 
genre de perfidie se trouve-t-il davantage dans le sexe faible que chez les hommes?"; "pourquoi dans le sexe si faible des femmes trouve-t-on davantage de sorcières que parmi les hommes?"'

Oeuvre de compilation, antiquus de par son contenu - car il reprend les opinions du passé, novus dans la disposition du texte, le Malleus Maleficarum aurait été mis en circulation à raison de plus de 30.000 exemplaires entre 1486 et 1669 à partir de maisons d'éditions de Franckfort, Nuremberg, Cologne, Spire, Bâle, Venise, Paris et Lyon, ce qui permettait à la majorité de ceux qui savaient lire d'avoir eu connaissance de l'ouvrage. ${ }^{10}$

Ce manuel a suscité bien des controverses parmi les historiens; les uns insistent sur le rôle important que le Malleus aurait joué dans l'intensification de la chasse aux sorcières. D'autres, au contraire, affirment que l'ouvrage ne constituait qu'une synthèse des idées véhiculées par la tradition et refusent d'établir un lien entre l'accroissement du nombre de procès de sorcellerie et la publication de ce manuel de démonologie. ${ }^{11}$

En ce qui nous concerne, nous insistons sur l'importance de ce manuel. Il peut être considéré comme une vaste synthèse qui, tout en se basant sur les textes de l'Ecriture, sur la tradition scolastique et sur les rapports de procès de sorcellerie, vise à persuader les juges et les prédicateurs de la réalité des phénomènes de sorcellerie et constitue un guide pratique utile lors des procès et condamnations des suspects. En plus d'être un dossier original des mentalités populaires de la vallée du Rhin au XVe siècle, il nous renseigne sur les tentatives effectuées afin de prévenir les mauvais sorts, de guérir les victimes, bref, de circonscrire le mal. Il représente une théorisation de la répression du mal qui ne sera appliquée qu' au XVI $\mathrm{X}^{\mathrm{e}}$ siècle.

Dans un monde mouvant, le Malleus laisse transparaître un malaise général qui se traduit par un essai de compréhension et d'interprétation des manifestations de la culture populaire.

L'une des caractéristiques essentielles du Malleus est d'affirmer que la "perversion hérétique" affecterait principalement les femmes. Il est donc indispensable de se pencher sur la vision des femmes telle qu'elle apparaît dans le manuel.

Le Malleus présente d'abord les femmes comme des êtres peu nuancés, incapables de tenir le juste milieu en fait de bonté et de malice. ${ }^{12}$ En plus d'être crédules, plus impressionnables, influençables, bavardes et perfides, elles sont "déficientes d'âme et de corps," inférieures aux hommes en ce qui concerne l'intelligence et la compréhension des choses spirituelles. ${ }^{13} \mathrm{La}$ raison de cette infériorité est attribuée à la nature même de la femme: "or, de cela, la raison naturelle, c'est qu'elle est plus charnelle que l'homme: on le voit de par ses multiples turpitudes." ${ }^{14}$ Cette déficience intellectuelle de la femme trouverait son origine, selon le Malleus dans une défectuosité apparue 
lors de la création de la première femme: “... puisqu'elle a été faite d'une côte courbe, c'est-à-dire d'une côte de la poitrine, tordue et comme opposée à l'homme. Il découle aussi de ce défaut que comme un vivant imparfait, elle déçoit toujours."15

Sources à l'appui, les auteurs s'efforcent d'illustrer la malice, la force et la redoutable violence qui caractérisent les envies, les rivalités, les jalousies, les vengeances et les haines féminines.

De plus, le Malleus nous présente les femmes comme étant envieuses, ${ }^{16}$ fragiles et portées à imaginer des choses extraordinaires, ${ }^{17}$ coquettes, querelleuses et peu dignes de foi car elles se laissent emporter par leurs inimitiés; aussi, on n'accordera qu'une confiance limitée à leurs assertions comme témoins lors d'un procès à moins qu'elles ne soient appuyées par les déclarations d'autres témoins, ${ }^{18}$ vu que leurs dépositions sont souvent faites par jalousie.

Si le Malleus insiste d'une part sur le fait que "pleurer, tisser et tromper sont le propre de la femme,"19 il dénonce la source de cette corruption de la femme: la concupiscence de la chair et la passion charnelle qui est insatiable et inassouvissable chez elle ${ }^{20}$ et qui la poussent à "s'abandonner aux vices" ou à "satisfaire ses passions dépravées."

L'optique peu nuancée du Malleus met donc en évidence la faiblesse de caractère, d'intelligence, de volonté et de mémoire de la femme qui, incapable de s'imposer par la force, le fait par le biais de la séduction, perturbant ainsi l'encadrement sociétaire de la sexualité. En plus d'être obsédé par ce caractère charnel de la femme, les auteurs du Malleus sont habités par le fantasme de la femme dangereuse, à la sexualité débordante, incontrôlable, diabolique, au pouvoir inexplicable, un peu magique, inspirant bien plus de la crainte que de l'émerveillement et nécessitant la mise en place de mécanismes de défense. Le Malleus présente donc la femme sous deux aspects principaux: elle est faible de caractère et dangereuse car corrompue par la passion charnelle. Ces deux caractéristiques seront d'une importance primordiale dans le lien qui sera établi entre la femme et la sorcellerie.

Cette vision négative de la femme telle qu'elle apparaît dans le Malleus n'est pas originale: elle est influencée par le courant misogyne traditionnel. En plus de certains auteurs de l'Antiquité c'est surtout l'exégèse de la Bible, les écrits des Pères de l'Eglise et la pensée des philosophes et théologiens médiévaux qui ont eu le plus grand ascendant sur les auteurs du Malleus.

Deux traditions s'opposent dans la vision de la femme présentée par l'Eglise: celle de la femme, cause de la chute de l'homme - Eve, et celle de la femme du Nouveau Testament, source et symbole de la rédemption de l'homme - Marie. Certains auteurs affirment que la vision négative de la femme est nettement prédominante dans l'exégèse médiévale. ${ }^{21}$ En ce qui concerne le Malleus c'est principalement cette vision négative qui l'emporte. 


\section{Lien féminité-sorcellerie}

Si l'on considère le rapport existant entre féminité et sorcellerie, on pourrait affirmer que, de tout temps, la femme a constitué une inquiétude pour l'homme. Certains auteurs émettent l'hypothèse que la femme, siège de forces obscures, se situant au dehors du rationnel, serait peut-être plus apte à deviner ce qui est caché, et à prédire l'avenir, étant dotée de pouvoirs magiques. ${ }^{22} \mathrm{D}$ 'autres insistent plus sur le rôle économique joué par la femme dans la plupart des sociétés anciennes: amenées à cultiver les espèces végétales, elles découvraient rapidement les vertus curatives de certaines plantes. Cet étroit contact avec la nature pouvait leur donner des pouvoirs inspirant à la fois la crainte et le respect. ${ }^{23}$ Symbole de vie et de mort, la femme enfantait et présidait aux rituels funéraires, transmettant oralement ses secrets de guérisseuse.

A certaines périodes elle occupa des fonctions religieuses importantes. ${ }^{24}$ Cependant, dans la tradition judéo-chrétienne, les femmes furent confinées à jouer un rôle passif. L'Eglise leur refusa le droit de prêcher, de présider aux offices et d'administrer les sacrements: exclues de la hiérarchie religieuse, elles ne purent vivre leur foi que dans l'enceinte des couvents ou des hôpitaux.

Il est donc possible d'affirmer que l'initiative des femmes au Moyen Age a trouvé un exutoire dans la sorcellerie. En outre de nombreux auteurs prétendent que la participation importante des femmes dans les mouvements hérétiques s'expliquerait en partie par le fait que les femmes y occupaient des fonctions plus valorisantes et plus satisfaisantes que celles proposées par l'Eglise officielle. ${ }^{25}$

De plus, la réputation de sorcière et lanceuse de sorts pouvait s'acquérir rapidement dans l'atmosphère des craintes incessantes et des malheurs quotidiens caractérisant la vie des petites communautés rurales. La sorcière était sorcière par le fait même que les membres de la communauté la percevaient comme telle, bien plus que par ses "pouvoirs," son éventuelle étrangeté ou ses agissements. Une valeur symbolique était octroyée par la collectivité aux gestes, aux paroles, aux regards et à la pensée de cette femme dont la personnalité, les connaissances et l'intuition hantaient l'imagination populaire. Aidée par le hasard et les coïncidences, on lui attribuait une faculté de réaliser ses volontés ainsi qu'une force mystérieuse lui donnant un pouvoir sur elle-même et sur son entourage, force identifiable au mana dont bénéficieraient certains individus. ${ }^{26}$ Toutefois, pour les juges, les théologiens, les démonologues et les inquisiteurs, tout ce qui n'était pas naturel relevait du miracle et de l'intervention divine. De même, tout sacré non officiel relevait du démoniaque.

Or, Satan, cherchant à saper la puissance du Créateur, trouvait des complices parmi les femmes. C'est ainsi que les auteurs du Malleus affirment que 


\section{8 / Renaissance and Reformation}

les femmes caractérisées par la faiblesse de leur intelligence, de leur volonté et de leur foi, plus portées aux vices que les hommes, constituent des acolytes de choix. Incapables de s'imposer par la raison et le contrôle personnel, elles cherchent à nuire par le biais de la séduction et de l'ensorcellement, abjurent plus facilement la foi et établissent un pacte avec Satan. ${ }^{27}$ De plus l'insatiabilité de leurs désirs les pousse à se livrer aux "turpitudes diaboliques des actes charnels avec les démons incubes,"28 turpitudes dénoncées à plusieurs reprises par le Malleus qui leur consacre d'ailleurs un chapitre entier. ${ }^{29}$

En établissant une complicité entre certaines femmes et Satan, les auteurs du Malleus essayaient de résoudre - consciemment ou non - le problème du mal: en diabolisant la femme, ils la rendaient désormais coupable des pires maux frappant les hommes et les bêtes, tout en méprisant sa sexualité et ses désirs.

Ayant clairement établi la nature diabolique du lien existant entre féminité et sorcellerie, le Malleus fournit des détails concernant les circonstances de l'ensorcellement et l'hommage rendu à Satan.

Selon le manuel, trois méthodes étaient utilisées par le diable pour attirer et séduire les innocents. Dans un premier cas, les démons profitaient des ennuis matériels encourus par les gens. L'aide était accordée en échange d'une certaine complicité avec le diable: le fidèle s'engageait à cracher par terre durant l'élévation du corps du Christ ou à refuser de confesser les péchés mortels. ${ }^{30}$ La seconde méthode était destinée aux femmes "plus adonnées aux passions et aux plaisirs." Le diable se mettait alors au service des désirs féminins. ${ }^{31}$ La troisième méthode de corruption se faisait par la voie de la tristesse et de la pauvreté et était destinée aux jeunes fiancées, amantes et concubines qui avaient été abandonnées par leur séducteur. ${ }^{32}$

Quelle que soit la méthode utilisée, on remarque que le Diable apparaît toujours dans un moment de désarroi: revers financiers, solitude ou détresse affective. Le serment de fidélité au diable peut se faire de deux manières: l'une solennelle, l'autre privée, la première étant de loin la plus importante. ${ }^{33}$ La plupart du temps, la Sorcière retire peu de bienfaits matériels de cette alliance et encore moins de plaisir; néanmoins, elle lui permet d'accomplir des maléfices et de lancer des sorts.

L'idée du pacte apparaît plus de trente fois dans le manuel et constitue à elle seule un chapitre entier. ${ }^{34}$ A aucun moment les auteurs du Malleus ne semblent émettre un doute concernant l'authenticité de l'hommage. Ils insistent sur les marques diaboliques laissées par Satan sur ses fidèles: elles sont incapables de pleurer et restent muettes sous les tortures, dotées du maléfice de taciturnité. 35

Cependant, le stéréotype du sabbat et de l'hommage n'atteint pas son niveau le plus élaboré dans le Malleus et nous sommes encore loin de ce que Mandrou appelle "la grande fête de l'imaginaire diabolique," du fameux sabbat des sorcières. ${ }^{36}$ Cependant, l'hommage rendu à Satan constitue une 
parodie jugée d'autant plus sacrilège par l'Eglise du fait que les participants étaient d'anciens chrétiens: c'était une apostasie systématique et organisée permettant la transgression des tabous et des interdits sexuels existant au niveau des villages ou des préceptes moraux de l'Eglise.

\section{Typologie des femmes-sorcières}

Malgré les nombreux exemples de maléfices mentionnés par le Malleus, il est difficile de faire une analyse exhaustive très précise des cas de sorcières mentionnées dans le manuel, ni du contexte social dans lequel elles évoluaient. En effet, l'auteur passe sous silence des informations concernant l'âge, la profession, le statut social de la sorcière, ainsi que des renseignements précis sur son lieu d'origine. Par contre, la nature du maléfice, les effets sur les victimes et les remèdes licites à employer sont largement décrits: la nature de l'outrage est donc plus importante que les détails personnels concernant le sorcier ou la sorcière.

La personnalité des femmes-sorcières du Malleus correspond plus ou moins à la personnalité des sorciers en général: ce sont des gens réputés tristes, peu sociables, souvent arrogants, susceptibles, colériques et nerveux, fréquemment impopulaires et rarement intégrés à part entière dans une communauté ${ }^{37}$ Leur ascendance sur les autres individus est troublante et une grande importance est attribuée à leurs yeux: ils dévisagent fixement les autres et peuvent nuire par la force de leur regard. ${ }^{38}$ Leurs gestes et leurs paroles sont perçus de façon symbolique par les témoins, ce qui leur confère un pouvoir particulier. ${ }^{39}$

Le Malleus donne peu de précisions concernant la profession des sorcières; certaines sont servantes, tenancières de bains et un grand nombre d'entre elles sont sages-femmes.

Dans un premier temps, la sorcière est acceptée par la communauté en raison des services qu'on attend d'elle. Cependant, elle reste à la merci d'un incident, d'une erreur. Il faut parfois peu de chose pour que la solidarité tacite des villageois et de cette "guérisseuse" se brise accidentellement: dès lors, la rumeur populaire attribuera à la sorcière toutes les malédictions se fréquentes en milieu rural.

L'existence du lien féminité-sorcellerie terrifie les auteurs du Malleus qui, dès lors, décrivent et prouvent la réalité des maléfices et des manifestations diaboliques opérées par l'intermédiaire des sorcières, afin de mieux légitimer la répression et le supplice final.

\section{La réalité des manifestations diaboliques et les méfaits des Maleficarum selon le Malleus}

Tous les exemples cités par les auteurs du Malleus - afin d'étayer leur démonstration - corroborent leur déclaration essentielle: ceux qui affirment le 
contraire de la foi aux sorciers sont hérétiques (Haeresis est maxima opera maleficarum non credere) ${ }^{40}$ Sprenger et Institoris n'ont pas choisi la voie du doute: ils reconnaissent avec certitude et vigueur l'existence réelle des maléfices. Or, cette opinion s'oppose catégoriquement au Canon Episcopi.

En effet, au tournant du $\mathrm{X}^{\mathrm{e}}$ siècle, les autorités religieuses - bien que reconnaissant implicitement l'importance de ces croyances - niaient la réalité des chevauchées nocturnes et condamnaient à l'exil les fidèles qui auraient foi dans ces inventions démoniaques. Résumer le Canon Episcopi consisterait donc à affirmer: haeresis est maxima opera maleficarum credere .

Rejetant cette opinion, les auteurs du Malleus affirment que nier la puissance du diable et la réalité des maléfices consisterait à ne pas reconnaître la permission et la puissance divine, ce qui irait à l'encontre de la foi. En effet, Dieu permettrait au Diable de faire le mal, sachant bien que son oeuvre divine ne pourrait jamais être perturbée au point d'être complètement détruite. Or, le libre arbitre de l'homme est capable du bien et du mal: les sorcières sont donc coupables aux yeux des inquisiteurs puisqu'elles ont établi volontairement un pacte avec les forces démoniaques. Elles sont d'autant plus coupables qu'elles font partie, selon le Malleus, d'une nouvelle hérésie des sorciers dont les caractères originaux sont: le pacte avec Satan, le reniement de la foi, la consécration totale aux démons, l'offrande d'enfants et les turpitudes charnelles effectuées avec les incubes et les succubes. En soutenant l'idée que cette hérésie était nouvelle, les auteurs du Malleus contournaient habilement la doctrine du Canon Episcopi, dernier obstacle à la répression légitime des sorcières.

Dès lors, n'ayant plus rien de commun avec les vieilles croyances folkloriques tolérées par l'Eglise dans le passé, cette nouvelle hérésie devait être réprimée.

La peur secrétée par l'insécurité et la montée du satanisme fut traduite - au niveau de l'élite religieuse - par le virulent discours des démonologues. Plus sensibles qu'autrefois à la permanence des forces du passé, ils opposèrent une interprétation religieuse de la sorcellerie à l'interprétation magique qu'en donnaient les populations. Le langage théologique du Malleus tenta de traduire et d'expliquer le langage magique: la "confusion sémantique" s'installa pendant plus de deux siècles entre l'intelligentsia et les représentants de la tradition syncrétiste du monde paysan. Les notions de sorcellerie (maleficium) allaient être mélangées aux notions de diabolisme: l'invocation et l'acte de sorcellerie étaient accomplis grâce à l'alliance diabolique. Véhiculé par le langage rigide des théologiens, cet entremêlement allait engendrer une démonologie au service du pouvoir.

Les auteurs du Malleus, persuadés de la réalité des oeuvres des sorciers, puisent abondamment dans les récits relatant les maléfices: témoignages recueillis personnellement, transmis par d'autres inquisiteurs, lus dans des manuels parus antérieurement, faits rapportés de bouche à oreille: tout sert à 
renforcer la certitude inébranlable des auteurs. Par leurs regards, leurs gestes, leurs paroles et leurs silences, les sorcières nuisent aux hommes et aux bêtes, provoquent des tempêtes et diverses catastrophes. Bien plus, de par leurs maléfices, elles attaquent directement le "coeur" de l'homme: l'être humain est donc menacé dans ses sentiments, sa raison, sa santé, sa puissance génitale et sa sexualité - ces deux derniers retenant particulièrement l'attention des deux inquisiteurs.

\section{Sorcellerie et natalité}

Il est établi qu'au Moyen Age, de nombreuses femmes pratiquaient la médecine: elles soignaient les blessures, guérissaient au moyen de potions et de remèdes et accouchaient les autres femmes. Au niveau du village, le rôle de la "femme-qui-soignait" était ambigu: elle pouvait guérir mais aussi provoquer la mort. De par ses connaissances elle exerçait un certain pouvoir au sein de la communauté et on lui reconnaissait souvent la capacité de contrôler la dose et le niveau de souffrance des humains; de par son rôle traditionnel, elle présidait à la naissance et à la mort: elle pouvait empêcher de naître, tuer à la naissance, forcer le nouveau-né à pousser ses premiers cris, prolonger l'agonie, retarder ou précipiter la mort. Dans un monde envahi par les pestes, les guerres, les épidémies et les famines récurrentes, elle pouvait facilement être perçue comme l'ultime refuge d'espérance face à la misère, à l'ignorance, à la maladie et à la mort.

Aussi-les auteurs du Malleus affirment que c'est parmi les sages-femmes que l'on trouve le plus de sorcières; selon eux, elles sont capables des pires maux: éviter que les femmes ne conçoivent, provoquer des avortements, dévorer l'enfant, l'offrir aux démons ${ }^{41}$ ou encore, le dépecer après la naissance: "Son office terminé, pendant qu'elle rentrait chez elle, au moment de franchir les portes de la ville, par hasard un bras d'enfant nouveau-né sortit de son tablier et tomba à terre. Ceux qui étaient aux portes le remarquèrent et après son passage s'en allèrent pour ramasser ce qu'ils pensaient être un morceau de viande. Mais regardant de plus près, ils reconnurent non pas un quelconque morceau de viande, mais un bras d'enfant avec ses articulations." 42

En insistant sur les sacrifices d'enfants, le Malleus ne fait pas preuve d'une grande originalité, ce thème apparaissant dans les manuels d'autres inquisiteurs. ${ }^{43}$ Il est certain que les soupçons à l'égard de la sage-femme ont dû se multiplier à la fin du Moyen Age: la sous-alimentation, les maladies, l'hygiène déplorable, les fatigues excessives et l'infanticide contribuaient certainement à provoquer une forte mortalité infantile. Un nombre anormal d'avortements et d'enfants mort-nés dans une région donnée permettaient à la population de suspecter avant tout la sage-femme. Dans une société où la fertilité occupait une place de premier ordre, les auteurs du Malleus compre- 
naient bien le rôle important de la sage-femme: elle pouvait contrôler la fécondité des couples et par là-même, celle des chrétiens.

Cependant, les pouvoirs maléfiques de la femme-sorcière ne se limitent pas au domaine de la fertilité: ils atteignent les êtres humains dans tous les aspects de leur sexualité.

\section{Sorcellerie et sexualité}

En effet, selon le Malleus, Dieu permet au diable un plus grand pouvoir maléfique sur les actes vénériens de l'homme que sur les autres actes pour deux motifs essentiels: la puissance génitale est frappée en raison du caractère honteux de l'acte et elle rappelle la chute du péché originel. Dans cette optique, la femme-sorcière - par le biais du pacte diabolique - peut assouvir sa vengeance et sa haine, en répandant de multiples maux tels que la stérilité, l'avortement, la frigidité, l'impuissance et la castration. Tout le domaine de la sexualité représente un danger pour les auteurs du Malleus; ainsi que le précise d'ailleurs Mircea Eliade, les théologiens étaient très conscients du pouvoir magique de la sexualitée ${ }^{44}$ que le judéo-christianisme avait démonisé. Aussi, théologiens et inquisiteurs se seraient imposés une ascèse rigide face à cette démonisation de la sexualité, austérité d'autant plus marquée qu'elle contrastait avec le relâchement de la vie instinctive des hommes et des artistes de la Renaissance. ${ }^{45}$

Aussi, l'attitude des auteurs du Malleus est, en grande partie, l'expression du refoulement sexuel d'une élite cléricale au service de la chasteté, leur peur individuelle ne reflétant que la peur et les fantasmes du groupe socio-culturel auquel ils appartiennent.

En conclusion, les sorcières du Malleus, en plus de tuer les enfants, empêchent la fécondité des couples par la confection de potions et l'absorption de certaines herbes destinées à éviter les naissances, ${ }^{46}$ frappent les hommes d'impuissance sexuelle, ${ }^{47}$ et déclenchent la haine ou l'amour fou dans leur coeur. ${ }^{48}$

\section{Conclusion}

Dans le cadre d'une société en crise, impuissante face aux multiples calamités et à la mort, envahie par des peurs eschatologiques, désemparée par la destructuration du monde rural et de la famille, caractérisée par un repli des populations sur les traditions et croyances du passé, le Malleus constitue une réaction de fuite en avant effectuée par la culture savante face à la remontée d'une culture populaire, jugée dangereuse et inférieure.

Le Malleus prouve la réalité des manifestations diaboliques et des maléfices des sorcières en dénonçant l'existence d'un pacte établi entre Satan et les sorcières, pacte autorisé par la permission divine et réalisable grâce à l'infériorité psychologique, morale et intellectuelle des femmes, et au carac- 
tère insatiable de leur sexualité qui en font les complices soumises des forces démoniaques.

Les crimes dont on accusait les femmes-sorcières ne présentaient pas un caractère original; en effet, à plusieurs reprises dans le passé, les pouvoirs politiques et religieux avaient taxé des groupes marginaux des accusations d'infanticide, de cannibalisme et d'orgies, que ce soient les premiers chrétiens du $\mathrm{II}^{\mathrm{e}}$ siècle, les Pauliciens d'Arménie au VIII ${ }^{\mathrm{e}}$ siècle, les Vaudois et Cathares des $\mathrm{XII}^{\mathrm{e}}$ et $\mathrm{XIII}^{\mathrm{e}}$ siècles: tous étaient perçus comme suspects et dangereux par le pouvoir qui voyait dans leurs rassemblements et dans leurs rites une possibilité de rébellion ou de compétition face à l'autorité. ${ }^{49}$

Ce type d'accusations se retrouve au niveau de la sorcellerie, accusations qui seraient restées sans lendemain si elles n'avaient pas été alimentées par le support tacite et les dénonciations des populations. La sorcière serait la victime expiatoire située au point de rencontre de la peur spontanée des populations et de la peur réfléchie de l'élite intellectuelle de l'époque. Au niveau du village, son importance et sa fonction étaient en compétition directe avec celle du prêtre et des autorités locales. Au sein des communautés, la lutte pour le pouvoir ne pouvait trouver de répit que par la canalisation de l'agressivité sur un bouc émissaire; dans le cas de la sorcière, seul le feu du bûcher était susceptible de purifier la société.

Université de Montréal

\section{Notes}

1 J. Delumeau, La Peur en Occident XIVe-XVIII siècles (Paris, 1978), p. 197.

2 J.B. Russel, Witchcraft in the Middle Ages (Ithaca, [1972], 1974), p. 269.

3 En ce qui concerne la poussée du satanisme et la prolifération des manuels, voir $R$. Mandrou, Magistrats et sorciers en France au XVII siècle (Paris, 1968), p. 89; F. Rapp, L'Eglise et la vie religieuse en Occident d la fin du Moyen Age (Paris, 1971), p. 161; N. Cohn, Europe's Inner Demons (Suffolk, 1975), p. 73.

4 La Bulle papale Summis desiderantes affectibus d'Innocent VIII (5 décembre 1484) est publiée dans Henri Institoris et Jacques Sprenger, Le Marteau des Sorcières présenté par A. Danet (Paris, 1973), p. 117-120.

5 Voir l'introduction de A. Danet, Le Marteau des Sorcières, p. 15.

6 Concernant la biographie de Jacques Sprenger, consulter Joseph Hansen, Quellen und Untersuchungen zur Geschichte des Hexenwahns und der Hexenverfolgung im Mittelalter (Bonn, 1901; réimp. Hildesheim, 1963), p. 395-404.

7 Consulter la biographie de Henri Institoris dans Joseph Hansen, op. cit., p. 380-395.

8 Voir Jean Goulet, Le Malleus Maleficarum: sa structure et ses lois de composition, Ph.D., Philosophie médiévale, Montréal, 1975, p. 46.

9 Le Marteau, I, question 6, p. 198.

10 Joseph Hansen a dénombré treize éditions qui auraient paru dans les années 1487-1520, et seize autres éditions entre 1574 et 1669. Voir J. Hansen, op. cit., p. 364. De son côté, A. Danet a repéré 34 éditions entre 1486 et 1669, voir A. Danet, Introduction du Marteau des Sorcières p. 17 et 18. 


\section{4 / Renaissance and Reformation}

11 Concernant ces controverses, voir J. Delumeau, op. cit., p. 50; J. Michelet, La Sorcière (Paris, 1966), p. 151; R. Mandrou, op. cit., p. 73 et p. 90; M. Summers, Introduction dans Malleus Maleficarum (London, 1951), XIV, cité par J. Goulet, Le Malleus Maleficarum, p. 34; H.C. Lea, Materials toward a History of Witchcraft, 3 vol. (New York, 1957), vol. 1, p. 337; E.W. Monter, European Witchcraft (New York, 1969), p. 55-56; R.H. Robbins, The Encyclopedia of Witchcraft and Demonology (New York, 1959), p. 337; H.C. Lea A History of Inquisition of the Middle Ages, réimp. 3 vol. (New York 1956), vol. 3, p. 544; H.R. Trevor-Roper, Religion, the Reformation and Social Change (Londres, 1 ere

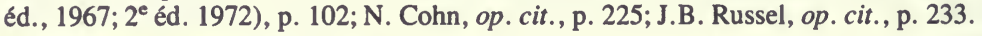

12 Le Marteau, I, question 6, p. 198.

13 Le Marteau, I, question 6, p. 201.

14 Le Marteau, I, question 6, p. 202.

15 Le Marteau, I, question 6, p. 203.

16 Le Marteau, II, Deuxième question principale, p. 440.

17 Le Marteau, II, Deuxième question principale, chapitre 1, p. 457.

18 Le Marteau, III, question 6, p. 547.

19 Le Marteau, III, question 15, p. 578.

20 Le Marteau, I, question 9, p. 208.

21 Joan Ferrante, Woman as Image in Medieval Literature, from the Twelfth century to Dante (New York, 1957), p. 1.

22 Marcel Mauss, Anthropologie et sociologie (Paris, 1967), p. 20.

23 Jean Delumeau, op. cit., p. 306.

24 Robert Briffault, The Mothers, 3 vol. (New York, 1927; $2^{\circ}$ éd., 1969), vol. II, p. 543.

25 Joan Ferrante, op. cit., p. 8; Harksen, Sibylle, La femme au Moyen Age (Leipzig, 1974), p. 37; Friedrich Heer, The Medieval World (New York, 1961), p. 203; J.B. Russel op. cit., p. 281.

26 Voir la définition du mana donnée par Codrington dans son ouvrage The Melanesians (1891) et cité par Georges Dumezil, La religion romaine archaique (Paris, 1966), p. 33: "C'est cette force qui produit tout ce qui est au-delà du pouvoir ordinaire des hommes, en dehors des règles communes de la nature. Il est présent dans l'atmosphère de la vie, s'attache aux personnes et aux choses, et se manifeste par des effets, qui ne peuvent être attribués qu'à son opération."

27 Le Marteau, I, question 6, p. 202.

28 Le Marteau, I, question 2, p. 161.

29 Le Marteau, $\mathrm{II}^{\mathrm{e}}$ partie, première question principale, chapitre 4: Comment les sorcières se livrent aux démons incubes, p. 337.

30 Le Marteau, II, Première question principale, chapitre 1, p. 310 et 311.

31 Le Marteau, II, Première question principale, chapitre 1, p. 311-312.

32 Le Marteau, II, Première question principale, chapitre 1, p. 313-314.

33 Le Marteau, II, Première question principale, chapitre 2, p. 318.

34 Le Marteau, II, Première question principale, chapitre 2, p. 316.

35 Le Marteau, II, Première question principale, chapitre 12, p. 402.

36 Robert Mandrou, op. cit., p. 80.

37 Voir un exemple dans Le Marteau, II, Première question principale, chapitre 3, p. 333; voir également Lucy Mair, La Sorcellerie (Paris, 1969), p. 40.

38 Le Marteau I, question 2, p. 156; II, Première question principale, chapitre 12, p. 402; II, Deuxième question principale, p. 445.

39 Le Marteau, II, question 6, p. 552; voir aussi Jeanne Favret-Saada, Les mots, la mort, les sorts, La sorcellerie dans le Bocage (Paris, 1977), p. 17.

40 Le Marteau, I, question 1, p. 143.

41 Le Marteau, I, question 11, p. 246.

42 Le Marteau, II, Première question principale, chapitre 13, p. 405. 


\section{Renaissance et Réforme/ 25}

43 Par exemple dans le manuel de Claude Tholosan, Ut magorem et maleficiorum errores, 1436, paragraphe 4, cité par Pierrette Paravy "A propos de la genèse médiévale des chasses aux sorcières: le traité de Claude Tholosan, juge dauphinois (vers 1436)" Mélanges de l'école française de Rome, Moyen Age Temps Modernes, MEFRM, tome q. 1, 1979, p. 333-379, p. 356: "dando sibi corpus et animan et alterum ex pueris suis, maxime primo genitum, quem sibi immolant et sacrifficant, genibus flexis, tenendo eum sub brachiis et humeris nudum et denum extingunt et post sepulturam exhumant et de eo, cum alii infrascriptis, faciunt pulverem."

44 Mircea Eliade, Occultisme, sorcellerie et modes culturelles (Paris, 1978), p. 122: "les théologiens étaient bien avertis du pouvoir magico-religieux de la sexualité, pouvoir capable de transformer des chrétiens en hérétiques sacrilèges et, pour finir, en démoniaques dangereux. Les taxer de démonolâtrie, n'était qu'une commode routine. Le fait décisif était que, imaginaires ou non, les orgies des sorciers, tout comme celles des hérétiques, pouvaient porter atteinte aux institutions sociales et religieuses."

45 Gregory Zilboorg, The Medical Man and the Witch during the Renaissance (Baltimore, 1935), p. 59.

46 Le Marteau, I, question 8, p. 223.

47 Le Marteau, II, Première question principale, chapitre 6, p. 355-356. Le Marteau, II, Première question principale, chapitre 7, p. 363-364.

48 Le Marteau, II, Première question principale, chapitre 10, p. 388.

49 Voir à ce sujet l'ouvrage de Norman Cohn: Europe's inner Demons. An Enquiry inspired by the great Witch-Hunt (Heinemann, pour Sussex University Press, 1975). 